\title{
FLORÍSTICA E ESTRUTURA DO COMPONENTE ARBUSTIVO-ARBÓREO DE UMA FLORESTA HIGRÓFILA DA BACIA DO RIO JACARÉ-PEPIRA, SP, BRASIL
}

\author{
Márcia C. M. Marques ${ }^{1}$ \\ Sandro Menezes Silva ${ }^{2}$ \\ Alexandre Salino ${ }^{3}$
}

Recebido em 25/08/2002. Aceito em 23/04/2003

\begin{abstract}
RESUMO - (Florística e estrutura do componente arbustivo-arbóreo de uma floresta higrófila da bacia do rio Jacaré-Pepira, SP, Brasil). As florestas higrófilas são formações ribeirinhas caracterizadas por ocorrerem em solo permanentemente encharcado e restritas a pequenos fragmentos junto a outros tipos vegetacionais. Neste trabalho caracterizaram-se a florística e a estrutura do componente arbustivo-arbóreo (plantas com DAP $\geq 5 \mathrm{~cm}$ ) de uma área de 0,36 ha de floresta higrófila localizada em Brotas ( $48^{\circ} 06^{\prime} \mathrm{W} 22^{\circ} 16^{\prime} \mathrm{S}, 470 \mathrm{~m} . \mathrm{s} . \mathrm{m}$.), Estado de São Paulo, usando-se método de parcelas (total de 24 parcelas). No total foram amostrados 735 indivíduos, distribuídos em 32 famílias e 51 espécies. As espécies que se destacaram na comunidade devido aos elevados valores de importância foram Calophyllum brasiliense Camb., Protium almecega L. Marchand, Podocarpus sellowii Klotzch., Tapirira guianensis Aubl. e Dendropanax cuneatum DC. Decne. \& Planch. O índice de diversidade de Shannon foi igual a 2,81 , valor pouco superior aos descritos para florestas semelhantes. Na comunidade, as espécies generalistas com relação ao encharcamento do solo e as de solo drenado contribuíram na riqueza total (juntas $62 \%$ do total de espécies amostradas), enquanto as espécies de solo encharcado tiveram maior contribuição na composição da dominância (66\% da dominância total) e densidade (67\% da densidade total) relativas. A diversidade de situações topográficas e a entrada de espécies da vegetação do cerrado adjacente permitiram que espécies com diferentes exigências hídricas se estabelecessem na área relativamente pequena da floresta e influenciaram fortemente a florística e estrutura da comunidade.
\end{abstract}

Palavras-chave - mata de brejo, inundação, floresta ciliar, fitossociologia, formação ribeirinha

\begin{abstract}
Floristics and structure of the shrub and the tree-layer of a swamp forest in Jacaré-Pepira river, Southeastern Brazil). Swamp forests occur in permanently flooded small forest fragments in southeastern Brazil. We studied the floristic composition and community structure (plants with $\mathrm{DBH} \geq 5 \mathrm{~cm}$ ) of a swamp forest in Brotas municipality ( $48^{\circ} 06^{\prime} \mathrm{W} 22^{\circ} 16^{\prime} \mathrm{S}$, $470 \mathrm{~m}$ high), São Paulo State, Brazil. A total of 735 individuals, 32 families, and 51 woody species were recorded in the area. The most important species were Calophyllum brasiliense
\end{abstract}

1 Departamento de Botânica, SCB, Universidade Federal do Paraná, C. Postal 19031, CEP 81531-970, Curitiba, PR, Brasil

2 Fundação O Boticário de Proteção à Natureza, Rua Gonçalves Dias, 225, CEP 80240-340, Curitiba, PR, Brasil (sandros@boticario.com.br)

3 Departamento de Botânica, ICB, Universidade Federal de Minas Gerais, C. Postal 486, CEP 30123-970, Belo Horizonte, MG, Brasil (salino@icb.ufmg.br)

Autor para correspondência: mmarques@ufpr.br 
Camb., Protium almecega L. Marchand, Podocarpus sellowii Klotzch., Tapirira guianensis Aubl. and Dendropanax cuneatum DC. Decne. \& Planch. The Shannon diversity index $(2,81)$ was greater than that of similar forests. With respect to soil humidity, the majority of species $(62 \%)$ are either generalists or prefer dry soils. However, dominant species ( $66 \%$ of total dominance) and species in high densities (67\% of total density) prefer waterlogged soils. Differences in soil topography and the strong influence of surrounding savanna guaranteed the occurrence of species with different soil humidity requirement and determined the floristics and structure of the swamp forest.

Key words - swamp forest, flooding, riparian forest, gallery forests, phytosociology

\section{Introdução}

As formações florestais que ocorrem ao longo de rios são condicionadas por série de fatores físicos, tais como relevo, profundidade do lençol freático e características do próprio rio (Rodrigues 1989; Rodrigues \& Shepherd 2000; Correia et al. 2001; Martins et al. 2001). Tais fatores estabelecem a freqüência e a duração das inundações, que por sua vez determinam a ocorrência ou não das espécies vegetais (Joly 1992). Como estes fatores ambientais são bastante variáveis entre as formações ribeirinhas, há grande heterogeneidade na estrutura e composição florística destas florestas (Leitão-Filho 1982; Nilsson et al.1988; Mantovani et al. 1989; Rodrigues \& Nave 2000).

Dentre as diferentes formações ribeirinhas, um tipo vegetacional bastante peculiar destacase por ocorrer em solo encharcado quase em caráter permanente e encontrar-se rodeado por vegetação estrutural e floristicamente diferenciada. É a chamada "floresta higrófila", "Mata de brejo" (Leitão-Filho 1982) ou "floresta estacional semidecidual ribeirinha com influência fluvial permanente" (Rodrigues 2000), a qual tem área de ocorrência bastante limitada. $\mathrm{O}$ fato destas florestas desempenharem papel importante na proteção de mananciais hídricos (Joly 1992; Marques 1994) tem feito com que os estudos florísticos e estruturais em tais locais fossem intensificados nos últimos anos (Torres et al. 1994; Ivanauskas et al. 1997; Costa et al. 1997; Toniato et al. 1998).

No Estado de São Paulo, as formações ribeirinhas encontram-se extremamente degra- dadas, situação esta causada principalmente pelo avanço das áreas de exploração agrícola (Camargo et al. 1971; Salvador 1987). Na bacia do rio Jacaré-Pepira, localizada na região central do Estado, a situação não é diferente. Fragmentos florestais de diferentes dimensões e graus de alteração distribuem-se ao longo da bacia, guardando algumas semelhanças florísticas e estruturais entre si (Salis et al. 1994; Metzger et al. 1997; Bernacci et al. 1998; Metzger et al. 1998). Nesta bacia, um projeto envolvendo prefeituras e pesquisadores da Universidade Estadual de Campinas foi desenvolvido com o intuito de preservar os remanescentes e recompor a vegetação onde fora removida (Joly et al. 2000). Neste contexto, o presente trabalho foi realizado em um remanescente de floresta higrófila na bacia do rio Jacaré-Pepira, em Brotas, SP e teve como objetivos: 1) avaliar a florística e a estrutura da fitocenose; 2) comparar a floresta em estudo com outras semelhantes no Estado de São Paulo, em termos qualitativos e quantitativos; 3) fornecer subsídios para o plano de recomposição ciliar regional.

\section{Material e métodos}

Área de estudo - O trabalho foi realizado em um fragmento de floresta higrófila com área total de aproximadamente 13ha, localizada próxima ao Viveiro Municipal de Brotas, SP $\left(48^{\circ} 06^{\prime} \mathrm{W}\right.$ $22^{\circ} 16^{\prime}$ S, $470 \mathrm{~m}$. s.m.). O local é atravessado por dois riachos, afluentes do córrego do Gouveia, um tributário da margem direita do rio JacaréPepira. O fragmento estudado tem formato alongado e estreito e, nas suas adjacências, 
encontram-se áreas de cultivo de laranja, além de pequenos remanescentes de Cerrado.

O clima da região é, segundo a classificação de Köppen, tropical sub-quente úmido, com temperatura média anual de $22^{\circ} \mathrm{C}$ e precipitação média de $1.421 \mathrm{~mm}$ (valores de 1961-1990) e estação seca bem marcada no período entre abril e setembro, quando ocorre deficiência hídrica do solo (Marques 1994).

O solo na área de estudo é hidromórfico, apresentando, em alguns locais, água superficial em caráter permanente. Análises preliminares mostraram que se trata de solo muito ácido $(\mathrm{pH}$ entre 4,1 e 5,1), com teores de fósforo, potássio, cálcio e magnésio muito baixos e teor de alumínio médio a alto. Os valores de $\mathrm{H}^{+} \mathrm{e}$ a capacidade de troca catiônica são altos e o volume de saturação em bases muito baixo. $\mathrm{Na}$ análise granulométrica, a classe de textura dos primeiros $80 \mathrm{~cm}$ é "limo-arenoso subclasse arenoso" e nas regiões mais profundas "limoarenoso-barrento subclasse areno-barrento" (Marques 1994).

Em levantamento topográfico realizado na área de estudo verificou-se que há diferenças de quase $5 \mathrm{~m}$ entre o local mais alto e o mais baixo da floresta (Marques 1994; Marques \& Joly 2000a). Entre estes extremos, uma diversidade de sítios com maior e menor nível topográfico se distribuem, refletindo na profundidade do lençol freático, o que cria um mosaico de situações de umidade no solo da floresta (entre 3\% e 100\%, segundo Marques 1994). A luz fotossinteticamente ativa (PAR) que atinge o interior da área de estudo varia entre 0 e $32 \%$, tornando o sub-bosque bem sombreado (Marques \& Joly 2000a).

A floresta é composta por três estratos definidos, sendo o superior em torno de $20 \mathrm{~m}$, formado principalmente por indivíduos de Calophyllum brasiliense, Protium almecega, Podocarpus sellowii, Tapirira guianensis e Pera obovata. No estrato médio, com aproximadamente $4 \mathrm{~m}$ de altura, são freqüentes indivíduos de Arecaceae (Geonoma sp.), Melastoma- taceae (Miconia sp.), Rubiaceae (Psychotria cf. barbiflora, Rudgea viburnoides), Myrtaceae (Myrcia spp.) e Cyatheaceae (Cyathea delgadii). O estrato inferior (40 a $50 \mathrm{~cm}$ ) é pouco expressivo e com predomínio de algumas pteridófitas herbáceas.

Amostragem e parâmetros fitossociológicos Uma retângulo de $45 \times 80 \mathrm{~m}$ (área total de 0,36 ha) foi delimitado transversalmente na região central do fragmento e dividido em 24 parcelas contíguas de $10 \times 15 \mathrm{~m}$. O levantamento fitossociológico foi realizado em janeiro/1992 e todos os indivíduos com DAP (diâmetro à altura do peito) $\geq 5 \mathrm{~cm}$ foram amostrados e tiveram a altura e a circunferência registrados. Amostras de material botânico dos indivíduos marcados foram coletadas e herborizadas seguindo metodologia usual (Fidalgo \& Bononi 1984) e identificadas com auxílio de literatura ou por comparação em herbário, sendo que na delimitação de famílias utilizou-se o sistema de classificação de Cronquist (1981). Os materiais que se encontravam férteis foram incorporados ao acervo dos herbários UPCB (Departamento de Botânica da Universidade Federal do Paraná) e UEC (Departamento de Botânica da Universidade Estadual de Campinas).

Avaliaram-se os parâmetros fitossociológicos, com base em Mueller-Dombois \& Ellenberg (1974) e calcularam-se o índice de diversidade de Shannon ( $\left.H^{\prime}\right)$ e a equabilidade (J), de acordo com Pielou (1975), utilizando-se o programa Fitopac (Shepherd 1988). Comparações com outras florestas da bacia do rio Jacaré-Pepira e com florestas higrófilas já estudadas no Estado de São Paulo foram feitas, utilizando-se o índice de similaridade específica de Sørensen (S) (Mueller-Dombois \& Ellenberg 1974).

As espécies levantadas foram classificadas de acordo com a preferência em relação à umidade do solo, de acordo com informações de estudos experimentais (Lobo-Faria 1998; Lobo \& Joly 1998; Marques \& Joly 2000b) e de trabalhos florísticos que abordaram a 
ocorrência preferencial de espécies em vegetação ribeirinha (Torres et al. 1994; Ribeiro et al. 1999). Assim, foram definidas espécies que preferem solo encharcado (normalmente tolerantes à inundação), espécies de solo drenado (normalmente intolerantes) e espécies generalistas (indiferentes em relação à umidade do solo). Com base nesta classificação e na análise fitossociológica, verificou-se a contribuição relativa de cada grupo na riqueza e estrutura (freqüência, densidade e dominância) da floresta.

\section{Resultados}

Foram amostrados 735 indivíduos na área de estudo, distribuídos em 32 famílias, 44 gêneros e 51 espécies, numa densidade total de 2042 ind.ha $^{-1}$. As famílias com maior número de indivíduos foram Burseraceae (144), Clusiaceae (133), Podocarpaceae (83), Magnoliaceae (47), Anacardiaceae (43) e Araliaceae (40). A maioria das famílias foi representada por apenas uma espécie (ex. Anacardiaceae, Araliaceae, Burseraceae, Clusiaceae, Magnoliaceae e Podocarpaceae), enquanto Fabaceae e Myrtaceae apresentaram os maiores números de espécies (5 espécies cada) (Tab. 1). Nove espécies $(4,6 \%)$ não puderam ser determinadas ao nível específico por não apresentarem estruturas reprodutivas que certificassem a identificação. $\mathrm{O}$ índice de diversidade $\left(\mathrm{H}^{\prime}\right)$ foi de 2,81 e a equabilidade ( $\mathrm{J})$ igual a 0,71 .

A distribuição das plantas, de acordo com o valor de importância (VI), mostra que 10 espécies destacar

am-se na comunidade, representando $75 \%$ do VI total (Tab. 2): Calophyllum brasiliense (com altos valores de dominância, densidade e freqüência), Protium almecega (freqüência, densidade e dominância), Podocarpus sellowii (densidade e dominância), Tapirira guianensis (freqüência e dominância), Dendropanax cuneatum (frequiência), Pera obovata (dominância), Talauma ovata (densidade), Copaifera langsdorfii (dominância), Hirtella hebeclada e Styrax pohlii (altas frequiências).

Considerando-se a preferência das espécies em relação à umidade do solo, a contribuição dos grupos para a composição dos parâmetros fitossociológicos foi distinta (Fig. 1). Enquanto as espécies generalistas e de solo drenado contribuíram principalmente com a riqueza (35\% da riqueza total) da comunidade, as espécies que preferem solo encharcado tiveram maior participação na densidade e dominância relativas (ambas 67\%). As espécies de solo drenado tiveram pequena participação na composição dos parâmetros fitossociológicos.

\section{Discussão}

A diversidade de situações topográficas, que se refletem no nível do lençol freático e, consequientemente, no grau de encharcamento do solo e a entrada de espécies da vegetação adjacente influenciaram fortemente a florística e a estrutura da floresta higrófila do ribeirão Gouveia, em Brotas. Tais condições permitiram que espécies com diferentes exigências hídricas (Tab. 1) se estabelecessem na área relativamente pequena da floresta (13ha).

Para compreender as variações florísticas entre formações ribeirinhas com influência fluvial permanente (florestas higrófilas) e com influência fluvial sazonal (florestas ciliares), dentro e fora de uma mesma bacia hidrográfica, comparou-se a área de estudo com outras formações florestais regionais (Tab. 3). É possível verificar que, ao nível de família, a área estudada apresenta riqueza maior que outras florestas higrófilas, e bastante próxima das florestas ciliares. As famílias que mais se destacaram no presente estudo são, em maioria, aquelas citadas como típicas das florestas higrófilas de São Paulo. Clusiaceae, Burseraceae, Anacardiaceae, Euphorbiaceae e Magnoliaceae, importantes famílias neste tipo florestal (Leitão-Filho 1982; Ivanauskas et al. 1997), representaram juntas $55 \%$ do total de indivíduos amostrados na área de estudo. No entanto, além dessas, Podocarpaceae e Araliaceae foram 
Tabela 1. Relação das espécies amostradas em uma floresta higrófila da bacia do rio Jacaré-Pepira em Brotas, SP e suas respectivas preferências ao grau de saturação hídrica do solo: $\mathrm{D}=$ solo drenado; $\mathrm{E}=$ solo encharcado, $\mathrm{G}=$ generalista, $\mathrm{ND}=$ não determinado.

\begin{tabular}{|c|c|c|c|}
\hline Família/Espécie & Preferência & Família/Espécie & Preferência \\
\hline ANACARDIACEAE & & MAGNOLIACEAE & \\
\hline Tapirira guianensis Aubl. & G & Talauma ovata A. St.-Hil. & $\mathrm{E}$ \\
\hline ANNONACEAE & & MALPIGHIACEAE & \\
\hline $\begin{array}{l}\text { Duguetia furfuracea (A. St.-Hil.) Benth \& } \\
\text { Hook }\end{array}$ & $\mathrm{D}$ & $\begin{array}{l}\text { Byrsonima intermedia Juss. } \\
\text { MELASTOMATACEAE }\end{array}$ & $\mathrm{D}$ \\
\hline Guatteria australis A. St.-Hil. & G & Miconia sp. & ND \\
\hline Xylopia aromatica (Lam.) Mart. & $\mathrm{D}$ & Trembleya parviflora (D. Don) Cogn. & $\mathrm{D}$ \\
\hline ARALIACEAE & & MELIACEAE & \\
\hline Dendropanax cuneatum (DC.) Decne. \& & E & Guarea macrophylla Vahl & E \\
\hline Planch. & & Trichilia pallens C. DC. & $\mathrm{E}$ \\
\hline BIGNONIACEAE & & MIMOSACEAE & \\
\hline Tabebuia sp. & ND & Acacia polyphylla A. DC. & $\mathrm{D}$ \\
\hline BORAGINACEAE & & MONIMIACEAE & \\
\hline Cordia sellowiana Cham. & G & Mollinedia sp. & ND \\
\hline BURSERACEAE & & MORACEAE & \\
\hline Protium almecega $\mathrm{L}$. Marchand & E & Ficus guaranitica Chodat & $\mathrm{G}$ \\
\hline $\begin{array}{l}\text { CAESALPINIACEAE } \\
\text { Conaifera } \text { lanosdorfii Desf }\end{array}$ & & Ficus obtusiuscula (Miq.) Miq. & $\mathrm{G}$ \\
\hline $\begin{array}{l}\text { Copaifera langsdorfil Dest. } \\
\text { CECROPIACEAE }\end{array}$ & $G$ & Moraceae 1 & ND \\
\hline Cecropia pachystachya Trecul & $\mathrm{G}$ & MYRISTICACEAE & \\
\hline CHRYSOBALANACEAE & & Virola sebifera Aubl. & $\mathrm{D}$ \\
\hline Hirtella hebeclada Moric. ex. A. DC. & $\mathrm{G}$ & MYRSINACEAE & \\
\hline CLUSIACEAE & & Rapanea umbellata (Mart.) Mez & G \\
\hline Calophyllum brasiliense Cambess. & E & MYRTACEAE & \\
\hline CUNONIACEAE & & Calyptranthes lucida Mart. ex A. DC. & G \\
\hline Lamanonia ternata Vell. & $\mathrm{E}$ & Campomanesia sp. & ND \\
\hline CYATHEACEAE & & Myrcia formosiana A. DC. & $\mathrm{G}$ \\
\hline Cyathea delgadii Sternb. & E & Myrcia sp. & ND \\
\hline EUPHORBIACEAE & & Psidium sp. & ND \\
\hline Pera obovata (Klotzsch) Baill. & G & PODOCARPACEAE & \\
\hline Sebastiania brasiliensis (L.) Spreng & $\mathrm{E}$ & Podocarpus sellowii Klotzch. & $\mathrm{E}$ \\
\hline FABACEAE & & PROTEACEAE & \\
\hline Andira cf. paniculata Benth. & G & Roupala montana Aubl. & $\mathrm{D}$ \\
\hline Machaerium aculeatum Raddi & $\mathrm{D}$ & RUBIACEAE & \\
\hline Machaerium acutifolium Vogel & $\mathrm{D}$ & Alibertia edulis A. Rich. & G \\
\hline Machaerium brasiliense Vogel & $\mathrm{D}$ & Ixora venulosa Benth. & $\mathrm{D}$ \\
\hline Platypodium cf. elegans Vogel & $\mathrm{D}$ & Rudgea viburnoides (Cham.) Benth. & $\mathrm{G}$ \\
\hline FLACOURTIACEAE & & SAPOTACEAE & \\
\hline Casearia sylvestris $\mathrm{Sw}$. & G & Pouteria laurifolia Radlk. & G \\
\hline LAURACEAE & & STYRACACEAE & \\
\hline Ocotea pulchella Mart. & G & Styrax pohlii A.DC. & $\mathrm{D}$ \\
\hline Lauraceae 1 & ND & VOCHYSIACEAE & \\
\hline Lauraceae 2 & ND & Vochysia tucanorum Mart. & $\mathrm{D}$ \\
\hline
\end{tabular}


Tabela 2. Parâmetros fitossociológicos das espécies arbustivo-arbóreas amostradas em uma floresta higrófila da bacia do rio Jacaré-Pepira em Brotas, SP, segundo a ordenação do VI, (FA = freqüência absoluta, FR = freqüência relativa, DA = densidade absoluta, $\mathrm{DR}=$ densidade relativa, $\mathrm{DoR}=$ Dominância relativa, $\mathrm{VC}=$ valor de cobertura, $\mathrm{VI}=$ valor de importância).

\begin{tabular}{|c|c|c|c|c|c|c|c|c|c|}
\hline Espécie & N. ind. & N. parcelas & FA & DA & FR & DR & DoR & $\mathrm{VC}$ & VI \\
\hline Calophyllum brasiliense & 137 & 22 & 91,67 & 380,56 & 8,43 & 18,64 & 23,47 & 42,11 & 50,53 \\
\hline Protium almecega & 144 & 23 & 95,83 & 400,00 & 8,81 & 19,59 & 18,62 & 38,21 & 47,02 \\
\hline Podocarpus sellowii & 83 & 12 & 50,00 & 230,56 & 4,60 & 11,29 & 12,68 & 23,97 & 28,57 \\
\hline Tapirira guianensis & 43 & 18 & 75,00 & 119,44 & 6,90 & 5,85 & 8,33 & 14,18 & 21,08 \\
\hline Dendropanax cuneatum & 40 & 18 & 75,00 & 111,11 & 6,90 & 5,44 & 4,58 & 10,02 & 16,92 \\
\hline Pera obovata & 26 & 14 & 58,33 & 72,22 & 5,36 & 3,54 & 7,04 & 10,57 & 15,94 \\
\hline Talauma ovata & 47 & 12 & 50,00 & 130,56 & 4,60 & 6,39 & 4,85 & 11,24 & 15,84 \\
\hline Copaifera langsdorfii & 18 & 12 & 50,00 & 50,00 & 4,60 & 2,45 & 6,15 & 8,60 & 13,20 \\
\hline Hirtella hebeclada & 19 & 10 & 41,67 & 52,78 & 3,83 & 2,59 & 2,62 & 5,21 & 9,04 \\
\hline Styrax pohlii & 22 & 11 & 45,83 & 61,11 & 4,21 & 2,99 & 1,47 & 4,47 & 8,68 \\
\hline Cyathea delgadii & 24 & 9 & 37,50 & 66,67 & 3,45 & 3,27 & 1,31 & 4,57 & 8,02 \\
\hline Moraceae 1 & 14 & 9 & 37,50 & 38,89 & 3,45 & 1,90 & 0,54 & 2,44 & 5,89 \\
\hline Rapanea umbellata & 11 & 9 & 37,50 & 30,56 & 3,45 & 1,50 & 0,36 & 1,85 & 5,30 \\
\hline Andira cf. paniculata & 7 & 7 & 29,17 & 19,44 & 2,68 & 0,95 & 0,53 & 1,48 & 4,16 \\
\hline Calyptranthes lucida & 9 & 5 & 20,83 & 25,00 & 1,92 & 1,22 & 0,30 & 1,53 & 3,44 \\
\hline Myrcia sp. & 7 & 4 & 16,67 & 19,44 & 1,53 & 0,95 & 0,41 & 1,37 & 2,90 \\
\hline Alibertia edulis & 6 & 4 & 16,67 & 16,67 & 1,53 & 0,82 & 0,32 & 1,13 & 2,67 \\
\hline Ixora venulosa & 4 & 4 & 16,67 & 11,11 & 1,53 & 0,54 & 0,50 & 1,04 & 2,57 \\
\hline Lamanonia ternata & 4 & 4 & 16,67 & 11,11 & 1,53 & 0,54 & 0,45 & 1,00 & 2,53 \\
\hline Trichilia pallens & 5 & 4 & 16,67 & 13,89 & 1,53 & 0,68 & 0,16 & 0,84 & 2,38 \\
\hline Roupala montana & 4 & 4 & 16,67 & 11,11 & 1,53 & 0,54 & 0,20 & 0,74 & 2,28 \\
\hline Cecropia pachystachya & 6 & 3 & 12,50 & 16,67 & 1,15 & 0,82 & 0,16 & 0,98 & 2,13 \\
\hline Pouteria laurifolia & 3 & 2 & 8,33 & 8,33 & 0,77 & 0,41 & 0,86 & 1,26 & 2,03 \\
\hline Cordia sellowiana & 4 & 3 & 12,50 & 11,11 & 1,15 & 0,54 & 0,32 & 0,87 & 2,02 \\
\hline Platypodium cf. elegans & 6 & 2 & 8,33 & 16,67 & 0,77 & 0,82 & 0,32 & 1,13 & 1,90 \\
\hline Virola sebifera & 3 & 3 & 12,50 & 8,33 & 1,15 & 0,41 & 0,33 & 0,74 & 1,89 \\
\hline Rudgea viburnoides & 3 & 3 & 12,50 & 8,33 & 1,15 & 0,41 & 0,08 & 0,49 & 1,64 \\
\hline Sebastiania brasiliensis & 4 & 2 & 8,33 & 11,11 & 0,77 & 0,54 & 0,17 & 0,71 & 1,48 \\
\hline Ficus guaranitica & 2 & 2 & 8,33 & 5,56 & 0,77 & 0,27 & 0,29 & 0,57 & 1,33 \\
\hline Xylopia aromatica & 2 & 2 & 8,33 & 5,56 & 0,77 & 0,27 & 0,25 & 0,52 & 1,29 \\
\hline Guarea macrophylla & 3 & 2 & 8,33 & 8,33 & 0,77 & 0,41 & 0,11 & 0,51 & 1,28 \\
\hline Vochysia tucanorum & 2 & 1 & 4,17 & 5,56 & 0,38 & 0,27 & 0,47 & 0,74 & 1,12 \\
\hline Tabebuia sp. 1 & 1 & 1 & 4,17 & 2,78 & 0,38 & 0,14 & 0,60 & 0,74 & 1,12 \\
\hline Machaerium aculeatum & 2 & 2 & 8,33 & 5,56 & 0,77 & 0,27 & 0,06 & 0,34 & 1,10 \\
\hline Psidium sp. & 2 & 2 & 8,33 & 5,56 & 0,77 & 0,27 & 0,06 & 0,33 & 1,10 \\
\hline Duguetia furfuracea & 1 & 1 & 4,17 & 2,78 & 0,38 & 0,14 & 0,28 & 0,41 & 0,80 \\
\hline Lauraceae 1 & 2 & 1 & 4,17 & 5,56 & 0,38 & 0,27 & 0,14 & 0,41 & 0,80 \\
\hline Casearia sylvestris & 2 & 1 & 4,17 & 5,56 & 0,38 & 0,27 & 0,08 & 0,35 & 0,73 \\
\hline Machaerium brasiliensis & 1 & 1 & 4,17 & 2,78 & 0,38 & 0,14 & 0,17 & 0,30 & 0,69 \\
\hline Acacia polyphylla & 1 & 1 & 4,17 & 2,78 & 0,38 & 0,14 & 0,06 & 0,20 & 0,58 \\
\hline Campomanesia sp. & 1 & 1 & 4,17 & 2,78 & 0,38 & 0,14 & 0,05 & 0,18 & 0,57 \\
\hline Myrcia formosiana & 1 & 1 & 4,17 & 2,78 & 0,38 & 0,14 & 0,03 & 0,17 & 0,55 \\
\hline Machaerium acutifolium & 1 & 1 & 4,17 & 2,78 & 0,38 & 0,14 & 0,03 & 0,17 & 0,55 \\
\hline Guatteria australis & 1 & 1 & 4,17 & 2,78 & 0,38 & 0,14 & 0,03 & 0,17 & 0,55 \\
\hline Mollinedia sp. 1 & 1 & 1 & 4,17 & 2,78 & 0,38 & 0,14 & 0,03 & 0,17 & 0,55 \\
\hline
\end{tabular}


Tabela 2 (continuação)

\begin{tabular}{|c|c|c|c|c|c|c|c|c|c|}
\hline Espécie & $\mathrm{N}$. ind. & N. parcelas & FA & DA & FR & DR & DoR & $\mathrm{VC}$ & VI \\
\hline Ocotea pulchella & 1 & 1 & 4,17 & 2,78 & 0,38 & 0,14 & 0,03 & 0,17 & 0,55 \\
\hline Trembleya parviflora & 1 & 1 & 4,17 & 2,78 & 0,38 & 0,14 & 0,03 & 0,17 & 0,55 \\
\hline Lauraceae 2 & 1 & 1 & 4,17 & 2,78 & 0,38 & 0,14 & 0,02 & 0,16 & 0,54 \\
\hline Byrsonima intermedia & 1 & 1 & 4,17 & 2,78 & 0,38 & 0,14 & 0,02 & 0,16 & 0,54 \\
\hline Miconia sp. 1 & 1 & 1 & 4,17 & 2,78 & 0,38 & 0,14 & 0,02 & 0,16 & 0,54 \\
\hline Ficus obtusiuscula & 1 & 1 & 4,17 & 2,78 & 0,38 & 0,14 & 0,02 & 0,15 & 0,53 \\
\hline
\end{tabular}

também muito importantes, com $17 \%$ dos indivíduos. Myrsinaceae, Annonaceae e Styracaceae que, segundo Leitão-Filho (1982) e Ivanauskas et al. (1997) são bem representativas em florestas higrófilas, apresentaram na área de estudo poucos indivíduos (5\% do total). Quanto ao número de espécies, a maior diversidade em Fabaceae e Myrtaceae segue padrões já descritos para outras formações ribeirinhas (Bernacci et al. 1998; Toniato et al. 1998). Por outro lado, Boraginaceae, Chrysobalanaceae, Cunoniaceae, Malpighiaceae, Monimiaceae e Vochysiaceae (todas

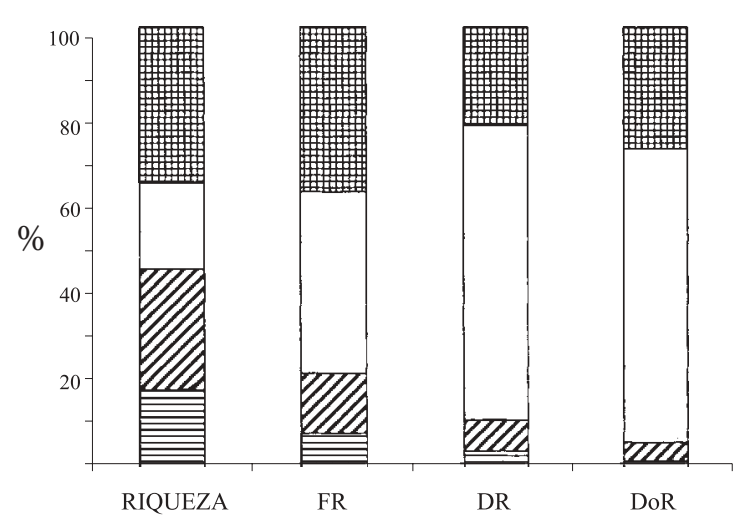

Figura 1. Distribuição percentual dos parâmetros fitossociológicos das espécies amostradas, agrupadas de acordo com a preferência ao grau de saturação hídrica do solo. RIQUEZA = percentagem da riqueza total de espécies; FR $=$ freqüência relativa, $\mathrm{DR}=$ densidade relativa, DoR = dominância relativa. 囵 Generalista; $\square$ Solo encharcado; Solo drenado; 目 Outros. representadas no presente estudo por apenas uma espécie), são famílias que não ocorreram nas demais florestas higrófilas.

Embora haja alguma semelhança entre as formações ribeirinhas com relação às famílias que mais se destacam, não há similaridade (baseado em parâmetros de Mueller-Dombois \& Ellenberg 1974) em termos de espécies entre a floresta estudada e outras áreas da região ( $S \leq 0,24$, Tab. 3). A dissimilaridade é verificada quando a comparação é feita com outras florestas higrófilas ou com florestas ciliares, até dentro da própria bacia do Jacaré-Pepira (Tab. 3). Mesmo comparando-se com um mesmo tipo vegetacional estudado também em Brotas (ribeirão do Onça, Costa et al. 1997), apenas $24 \%$ das espécies são comuns entre as áreas. A grande heterogeneidade florística entre formações ribeirinhas, mesmo em se tratando de uma mesma microbacia hidrográfica, já foi reportada anteriormente (Rodrigues \& Nave 2000; Silva Júnior et al. 2001). A diversidade ambiental nas áreas ciliares determina condição ecotonal ocupada por mosaico de tipos vegetacionais (Rodrigues \& Nave 2000) diretamente influenciados, entre outros, pela proximidade geográfica com outras fitocenoses (Ivanauskas et al. 1997; Silva Júnior et al. 2001). No caso floresta estudada, fragmentos de florestas estacionais semideciduais e cerrado que ocorrem nas proximidades da área contribuem com várias espécies, o que aumentam as diferenças florísticas com outras áreas de floresta higrófila. 
Tabela 3. Comparação entre a floresta estudada e outras áreas florestais ribeirinhas da região central do Estado de São Paulo, quanto à riqueza de famílias $\left(\mathrm{R}_{\text {famílias }}\right)$, similaridade florística de $\mathrm{S} \emptyset$ rensen $\left(\mathrm{S}_{\text {espécies }}\right)$ e índice de diversidade $\left(\mathrm{H}^{\prime}\right)$.

\begin{tabular}{|c|c|c|c|c|c|}
\hline Localidade & Tipo florestal & $\mathrm{R}_{\text {famílias }}$ & $\mathrm{S}_{\text {espécies }}$ & $\mathrm{H}^{\prime}$ & Referência* \\
\hline \multicolumn{6}{|l|}{ bacia do rio Jacaré-Pepira } \\
\hline Brotas - ribeirão Gouveia & floresta higrófila & 32 & - & 2,81 & 1 \\
\hline Brotas - ribeirão do Onça & floresta higrófila & 22 & 0,24 & 2,52 & 2 \\
\hline região de Brotas Fragmento F21 & floresta ciliar & 40 & 0,19 & 4,30 & 3 \\
\hline Brotas - rio Jacaré-Pepira & floresta ciliar & 32 & 0,07 & 3,06 & 4 \\
\hline região de Brotas Fragmento F31 & floresta ciliar & 34 & 0,14 & 3,60 & 3 \\
\hline região de Brotas Fragmento F11 & floresta ciliar & 26 & 0,01 & 3,08 & 3 \\
\hline \multicolumn{6}{|l|}{ Outras bacias } \\
\hline Itatinga & floresta higrófila & 28 & 0,22 & 2,75 & 5 \\
\hline Campinas & floresta higrófila & 23 & 0,21 & 2,45 & 6 \\
\hline Campinas & floresta higrófila & 29 & 0,21 & 2,80 & 7 \\
\hline Itirapina & floresta ciliar & 32 & 0,04 & 3,60 & 8 \\
\hline
\end{tabular}

\footnotetext{
* Referência: 1) presente estudo, 2) Costa et al. 1997, 3) Metzger et al. 1998 e Bernacci et al. 1998 (dentre os 15 fragmentos estudados pelos autores foram escolhidos para comparação os 3 que apresentavam área superior a 10ha), 4) Salis et al. 1994, 5) Ivanauskas et al. 1997, 6) Torres et al. 1994, 7) Toniato et al. 1998, 8) Kotchetkoff-Henriques \& Joly 1994.
}

Apesar das formações ribeirinhas em geral serem bem diversas em termos florísticos (Rodrigues \& Nave 2000), aquelas sujeitas à maior frequiência do alagamento tendem a apresentar menores riqueza e diversidade que as pouco inundadas (Metzger et al. 1998). Segundo Leitão-Filho (1982), as florestas higrófilas são menos diversas que as demais formações ribeirinhas. Isto se deve ao fato da inundação restringir a ocorrência de muitas espécies que não toleram a hipoxia do solo (Joly 1991; Marques et al. 1996). Na área estudada verificou-se índice de diversidade (H') ligeiramente superior aos já observados em florestas higrófilas, porém muito inferiores aos de florestas ciliares da região (Tab. 3). Esta situação intermediária deve-se, principalmente, à heterogeneidade em termos de umidade do solo da área de estudo, que propiciou a ocorrência de várias espécies típicas do cerrado e de florestas estacionais semideciduais, elevando a riqueza de espécies e famílias. Provavelmente o fato das formações ribeirinhas serem normalmente estreitas e conectadas através de pequenos corredores com outras áreas florestadas (Metzger et al. 1997) facilitou a entrada e a contribuição da vegetação adjacente na floresta higrófila de Brotas.

O total de $47 \%$ das espécies levantadas no presente estudo já havia sido descrito como ocorrente em florestas higrófilas (Torres et al. 1994; Ivanauskas et al. 1997; Costa et al. 1997; Toniato et al. 1998). Cecropia pachystachia, Tapirira guianensis, Copaifera langsdorfii, Calophyllum brasiliense e Casearia sylvestris, as quais foram amostradas na área de Brotas, estão entre as espécies de maior ocorrência em formações ribeirinhas (Rodrigues \& Nave 2000). No entanto, $53 \%$ do total de espécies amostradas ainda não havia sido listado para florestas higrófilas. Muitas destas são espécies comuns nos remanescentes de cerrado da região (Byrsonima intermedia, Duguetia furfuracea, Xylopia aromatica, Platypodium cf. elegans, Roupala montana, Copaifera langsdorfii, Vochysia tucanorum, Ocotea pulchella) ou ocorrem em florestas estacionais semideciduais do Estado (Cordia sellowiana, Hirtella hebeclada, Lamanonia ternata, Sebastiania brasiliensis, Acacia polyphylla, Andira cf. paniculata, Machaerium acutifolium, M. brasiliensis, Casearia sylvestris, Trembleya 
parviflora, Trichilia pallens, Ficus guaranitica, F. obtusiuscula, Virola sebifera, Calyptrantes lucida, Myrcia formosiana, Alibertia edulis, Ixora venulosa, Rudgea viburnoides). A influência da vegetação do cerrado e das florestas estacionais semideciduais em formações ribeirinhas é resultado, entre outros aspectos, das progressões e regressões pretéritas das formações vegetacionais, as quais ficam patentes nos estudos florísticos recentes (Oliveira-Filho \& Ratter 2000).

A ocorrência de espécies de outras formações vegetacionais em florestas higrófilas foi também abordada por Torres et al. (1994) e Ivanauskas et al. (1997) que classificaram as espécies ocorrentes neste tipo florestal em dois principais grupos. O primeiro é formado por espécies peculiares da floresta higrófila e, o segundo, pelas complementares, geralmente oriundas da vegetação adjacente. Considerandose que a maior parte das espécies da floresta estudada não havia sido citada para este tipo vegetacional, percebe-se que a contribuição das espécies complementares na florística da floresta estudada é muito expressiva.

As espécies com maiores VI são as mesmas já citadas para outras florestas higrófilas, embora haja pequena variação na ordenação das mesmas. Calophyllum brasiliense, Protium almecega e/ou Tapirira guianensis, respectivamente $1^{\mathrm{a}}, 2^{\mathrm{a}}$ e $4^{\mathrm{a}}$ posições no presente trabalho, estão sempre entre as primeiras espécies em ordem de VI (Costa et al. 1997). Por outro lado, Podocarpus sellowii, $3^{a}$ posição em VI em Brotas, somente ocorreu em Itatinga (Ivanauskas et al. 1997). Tais resultados corroboram a afirmação de Toniato et al. (1998) de que, muito mais que similaridade florística, as florestas higrófilas são semelhantes em relação à densidade de indivíduos das espécies comuns, o que as diferencia das demais formações ribeirinhas.

A estrutura da floresta higrófila fica facilmente compreendida quando se avalia a contribuição das espécies quanto à preferência ao tipo de solo. Enquanto espécies generalistas e de solo drenado aumentam a riqueza da floresta, as espécies de solo encharcado são importantes principalmente na definição da dominância e densidade. Poucas são as espécies reconhecidamente tolerantes à situação de solo encharcado. Ajustes envolvendo o metabolismo respiratório das raízes (Lobo \& Joly 1998), a germinação (Lieberg \& Joly 1993; Lobo \& Joly 1996; Marques \& Joly 2000b), a fenologia (Lobo-Faria 1998) e a morfologia de raízes e caules (Pimenta et al. 1998) condicionam a ocorrência de número reduzido de espécies. Experimentos demonstraram que plantas de Calophyllum brasiliense, Protium almecega, Talauma ovata e Tapirira guianensis crescem normalmente em solo inundado (Lobo-Faria 1998; Marques \& Joly 2000b). São espécies amplamente distribuídas nos neotrópicos, porém pontualmente localizadas em zonas sujeitas à inundação constante (Lobo \& Joly 2000) e além delas, algumas outras espécies suportam tal condição do solo (Ferreira \& Ribeiro 2001). No entanto, apesar de contribuírem pouco para a riqueza das florestas higrófilas, as espécies tolerantes ao encharcamento do solo definem a dominância e a densidade neste tipo vegetacional.

Segundo Correia et al. (2001) que consideraram florestas de galeria em meio à vegetação do cerrado, a concentração de biomassa ao longo de cursos d'água tem papel importante na conservação de nutrientes essenciais no ecossistema. Portanto, se considerar-se que as espécies de solo encharcado definem a dominância das florestas higrófilas, poder-se-ia considerá-las importantes na função de retenção de nutrientes no ecossistema. Por outro lado, espécies generalistas e as de solo drenado teriam chances de estabelecimento proporcional ao número de microsítios com solo melhor drenado disponíveis. Na floresta estudada, apesar de grande parte da área apresentar depressões e lençol freático superficial, as variações topográficas permitem a existência de muitos locais com solo de drenagem rápida (Marques \& Joly 
2000a). Devido à proximidade e possível entrada de diásporos das formações vizinhas, espécies tipicamente de solo drenado ou mesmo as generalistas são capazes de estabelecer-se.

Em conclusão, pode-se dizer que, apesar dos conhecimentos até agora acumulados sobre as florestas higrófilas de São Paulo indicarem formações aparentemente homogêneas, no que se refere à baixa diversidade e às poucas alterações florísticas no elenco de espécies comuns, variações ocorrem. A natureza fragmentada destas florestas, em decorrência da localização pontual em solos encharcados, ou da supressão de áreas florestais no Estado, a efetiva contribuição da flora adjacente, além das particularidades ambientais (principalmente número e distribuição de sítios mais ou menos inundados) são fatores que contribuem para a heterogeneidade florística nestas formações.

\section{Agradecimentos}

Ao Prof. Dr. Carlos Alfredo Joly, da UNICAMP, pelas facilidades oferecidas para o desenvolvimento do projeto; à Marília Borgo, pela confirmação de algumas espécies; ao James J. Roper, pela revisão do Abstract; à Prefeitura Municipal de Brotas (SP), pela permissão em trabalhar na área.

\section{Referências bibliográficas}

Bernacci, L. C.; Goldenberg, R. \& Metzger, J. P. 1998. Estrutura florística de 15 fragmentos florestais ripários da bacia do rio Jacará-Pepira (SP). Naturalia 23: 23-54.

Camargo, J. C. G.; Cesar, A. L.; Gentil, J. P.; Pinto, S. A. F. \& Tropmair, H. 1971. Estudo fitogeográfico da vegetação ciliar do rio Corumbataí, SP. Biogeografia 3: 1-14.

Correia, J. R.; Haridasan, M.; Reatto, A.; Martins, E. S. \& Walter, B. M. T. 2001. Influência de fatores edáficos na distribuição de espécies arbóreas em Matas de Galeria na região do Cerrado: uma revisão. Pp. 51-76. In J. F. Ribeiro; C. E. L. Fonseca \& J. C. Souza-Silva (eds.). Cerrado: caracterização e recuperação de Matas de Galeria. Embrapa, Planaltina.
Costa, F. R. C.; Schlittler, F. H. M.; Cesar, O. \& Monteiro, R. 1997. Aspectos florísticos e fitossociológicos de um remanescente de Mata de brejo no município de Brotas, SP. Arquivos de Biologia e Tecnologia 40(2): 263-270.

Cronquist, A. 1981. An integrated system of classification on flowering plants. Columbia University Press, New York.

Ferreira, J. N. \& Ribeiro, J. F. 2001. Ecologia da inundação em Matas de galeria. Pp. 425-451. In J. F. Ribeiro; C. E. L. Fonseca \& J. C. Souza-Silva (eds.). Cerrado: caracterização e recuperação de Matas de Galeria. Embrapa, Planaltina.

Fidalgo, O. \& Bononi, V. L. R. 1984. Técnicas de coleta, preservação e herborização de material botânico. Manual n. 4. Instituto de Botânica, São Paulo.

Ivanauskas, N. M.; Rodrigues, R. R. \& Nave, A. G. 1997. Aspectos ecológicos de um trecho de Floresta de brejo em Itatinga, SP: florística, fitossociologia e seletividade de espécies. Revista Brasileira de Botânica 20(2): 139-153.

Joly, C. A. 1991 Flooding tolerance in tropical trees. Pp. 23-34. In M. B. Jackson, D. D. Davies \& H. Lambers (eds.). Plant life under oxygen deprivation: ecology, physiology and biochemistry. SBP Academic Publishing, The Hague.

Joly, C. A. 1992. Biodiversity of gallery forest and its role in soil stability in the Jacaré-Pepira water, state of São Paulo, Brazil. Pp. 40-66. In A. Jensen (ed.). Ecotones at the river basin scale global land/ water interactions. Proceedings of ecotones regional workshop. UNESCO/MAB, Barmera.

Joly, C. A.; Spigolon, J. R.; Lieberg, S. A.; Salis, S. M.; Aidar, M. P. M.; Metzger, J. P. W.; Zickel, C. S.; Lobo, P. C.; Shimabukuro, M. T.; Marques, M. C. M. \& Salino, A. 2000. Projeto Jacaré-Pepira - o desenvolvimento de um modelo de recomposição da mata ciliar com base na florística regional. Pp. 271-287. In R. R. Rodrigues \& H. F. Leitão-Filho (eds.). Matas ciliares: conservação e recuperação. Edusp/Fapesp, São Paulo.

Kotchetkoff-Henriques, O. \& Joly, C. A. 1994. Estudo florístico e fitossociológico em uma mata mesófila semidecídua da Serra do Itaqueri, Itirapina, Estado de São Paulo, Brasil. Revista Brasileira de Biologia 54(3): 477-487.

Leitão Filho, H. F. 1982. Aspectos taxonômicos das florestas do Estado de São Paulo. Silvicultura em São Paulo 16A: 197-206. 
Lieberg, S. A. \& Joly, C. A. 1993. Inga affinis DC (Mimosaceae): germinação e tolerância de plântulas à submersão. Revista Brasileira de Botânica 16: 175-179.

Lobo, P. C. \& Joly, C. A. 1996. Ecofisiologia da germinação de sementes de Talauma ovata St. Hil. (Magnoliaceae), uma espécie típica de matas de brejo. Revista Brasileira de Botânica 19: 35-40.

Lobo, P. C. \& Joly, C. A. 1998. Tolerance to hipoxia and anoxia in neotropical tree species. Pp. 137-156. In F. R. Scarano \& A. C. Franco (eds.). Ecophysiological strategies of xerophytic and amphibious plants in the neotropics. Series Oecologia Brasiliensis. vol IV. PPGE-UFRJ, Rio de Janeiro.

Lobo, P. C. \& Joly, C. A. 2000. Aspectos ecofisiológicos da vegetação de mata ciliar do sudeste do Brasil. Pp. 143-157. In R. R. Rodrigues \& H. F. LeitãoFilho (eds.). Matas ciliares: conservação e recuperação. Edusp/Fapesp, São Paulo.

Lobo-Faria, P. C. 1998. Estratégias adaptativas de espécies arbóreas típicas de ambiente com solo hidricamente saturado: uma abordagem morfológica, bioquímica e ecofisiológica. Tese de Doutorado. Universidade Estadual de Campinas, Campinas.

Mantovani, W.; Rossi, L.; Romaniuc Neto, S.; AssadLudwig, I. Y.; Wanderley, M. G. L.; Melo, M. M. R. F. \& Toledo, C. B. 1989. Estudos fitossociológicos das áreas de mata ciliar em Mogi-Guaçu, SP, Brasil. Pp. 235-267. In L. M. Barbosa (ed.). Anais do Simpósio sobre mata ciliar. Fundação Cargil, Campinas.

Marques, M. C. M. 1994. Estudos auto-ecológicos do guanandi (Calophyllum brasiliense Camb. Clusiaceae) em uma mata ciliar do município de Brotas, SP. Dissertação de Mestrado. Universidade Estadual de Campinas, Campinas.

Marques, M. C. M.; Pimenta, J. A. \& Colli, S. 1996. Aspectos do metabolismo de Cedrela fissilis Vell. e Anadenanthera colubrina (Vell.) Bren. submetidas a diferentes regimes hídricos. Arquivos de Biologia e Tecnologia 39(2): 385-392.

Marques, M. C. M. \& Joly, C. A. 2000a. Estrutura e dinâmica de uma população de Calophyllum brasiliense Camb. em Floresta higrófila do sudeste do Brasil. Revista Brasileira de Botânica 23(1): 107-112.

Marques, M. C. M. \& Joly, C. A. 2000b. Germinação e crescimento de Calophyllum brasiliense Camb. (Clusiaceae), uma espécie típica de florestas inundadas. Acta Botanica Brasilica 14(1): 133-120.
Martins, E. S.; Reatto, A. \& Correia, J. R. 2001. Fatores ambientais que controlam as paisagens das Matas de Galeria no bioma Cerrado: exemplos e hipótese. Pp. 79-111. In J. F. Ribeiro; C. E. L. Fonseca \& J. C. Souza-Silva (eds.). Cerrado: caracterização e recuperação de Matas de Galeria. Embrapa, Planaltina.

Metzger, J. P.; Bernacci, L. C. \& Goldenberg, R. 1997. Pattern of tree species diversity in riparian forest fragments with different widths (SE Brazil). Plant Ecology 133: 135-152.

Metzger, J. P.; Goldenberg, R. \& Bernacci, L. C. 1998. Diversidade e estrutura de fragmentos de mata de várzea e de mata mesófila semidecídua submontana do rio Jacaré-Pepira (SP). Revista Brasileira de Botânica 21(3): 321-330.

Mueller-Dombois, D. \& Ellenberg, H. 1974. Aims and methods of vegetation ecology. John Wiley, New York.

Nilsson, C.; Grelsson, G.; Johansson, M. \& Sperens, U. 1988. Can rarity and diversity be predicted in vegetation along river banks? Biological Conservation 44: 201-212.

Oliveira-Filho, A. T. \& Ratter, J. A. 2000. Padrões florísticos das matas ciliares da região do Cerrado e a evolução das paisagens do Brasil central durante o quaternário tardio. Pp. 73-89. In R. R. Rodrigues \& H. F. Leitão-Filho (eds.). Matas ciliares: conservação e recuperação. Edusp/Fapesp, São Paulo.

Pielou, E. C. 1975. Ecological diversity. WieleyInterscience, New York.

Pimenta, J. A.; Bianchini, E. \& Medri, M. E. 1998. Adaptations to flooding by tropical trees: morphological and anatomical modifications. Pp. 157-176. In F. R. Scarano \& A. C. Franco (eds.). Ecophysiological strategies of xerophytic and amphibious plants in the neotropics. Series Oecologia Brasiliensis. vol IV. PPGE-UFRJ, Rio de Janeiro.

Ribeiro, J. F.; Walter, B. M. T. \& Fonseca, C. E. L. 1999. Ecossistemas de matas ciliares. Pp. 12-25. In Simpósio Mata Ciliar: ciência e tecnologia. Anais. CEMIG/UFLA, Lavras.

Rodrigues, R. R. 1989. Análise estrutural das formações ripárias. Pp. 99-119. In L. M. Barbosa (ed.). Anais do Simpósio sobre mata ciliar. Fundação Cargil, Campinas.

Rodrigues, R. R. 2000. Uma discussão nomenclatural das Formações Ciliares. Pp. 91-99. In R. R. Rodrigues \& H. F. Leitão-Filho (eds.). Matas ciliares: conservação e recuperação. Edusp/ Fapesp, São Paulo. 
Rodrigues, R. R. \& Shepherd, G. J. 2000. Fatores condicionantes da vegetação ciliar. Pp. 101-107. In R. R. Rodrigues \& H. F. Leitão-Filho (eds.). Matas ciliares: conservação e recuperação. Edusp/Fapesp, São Paulo.

Rodrigues, R. R. \& Nave, A. A. 2000. Heterogeneidade florística das Matas Ciliares. Pp. 45-71. In R. R. Rodrigues \& H. F. Leitão-Filho (eds.). Matas ciliares: conservação e recuperação. Edusp/ Fapesp, São Paulo.

Salis, S. M.; Tamashiro, J. Y. \& Joly, C. A. 1994. Florística e fitossociologia do estrato arbóreo de um remanescente de mata ciliar do rio JacaréPepira, Brotas, SP. Revista Brasileira de Botânica 17(2): 93-103.

Salvador, J. L. G. 1987. Considerações sobre matas ciliares e a implantação de reflorestamentos mistos nas margens de rios e reservatórios. CESP, São Paulo.
Shepherd, G. J. 1988. Fitopac - versão preliminar. Manual do usuário. Departamento de Botânica, Instituto de Biologia, Universidade Estadual de Campinas, Campinas.

Silva Júnior, M. C.; Felfili, J. M.; Walter, B. M. T.; Nogueira, P. E.; Rezende, A. V.; Morais, R. O. \& Nóbrega, M. G. G. 2001. Análise da flora de matas de Galeria no Distrito Federal: 21 levantamentos. Pp. 143-191. In J. F. Ribeiro; C. E. L. Fonseca \& J. C. Souza-Silva (eds.). Cerrado: caracterização e recuperação de Matas de Galeria. Embrapa, Planaltina.

Toniato, M. T. Z.; Leitão-Filho, H. F. \& Rodrigues, R. R. 1998. Fitossociologia de um remanescente de Floresta higrófila (Mata de brejo) em Campinas, SP. Revista Brasileira de Botânica 21(2): 197-210.

Torres, R. B.; Matthes, L. A. F. \& Rodrigues, R. R. 1994. Florística e estrutura do componente arbóreo de mata de brejo em Campinas, SP. Revista Brasileira de Botânica 17(2): 189-194. 